\title{
Multiscale study of influence of interfacial decohesion on piezoresistive of graphene/polymer nanocomposites
}

\author{
Xiaoxin Lu ${ }^{1,2}$, Fabrice Detrez ${ }^{2}$, Julien Yvonnet ${ }^{2}$, Jinbo Bai ${ }^{1}$ \\ 1 Université Paris Saclay, Laboratoire de Mécanique des Sols, Structures et \\ Matériaux, UMR 8579 CNRS, 8-10 rue Joliot Curie, 91190 Gif-sur-Yvette, France \\ ${ }^{2}$ Université Paris-Est, Laboratoire de Modélisation et Simulation Multi Echelle, \\ UMR 8208 CNRS, 5 Boulevard Descartes, 77454 Marne-la-Vallée Cedex 2, France \\ E-mail: fabrice.detrez@u-pem.fr, jinbo.bai@ecp.fr
}

October 2018

\begin{abstract}
A multiscale strategy is proposed to study the role of interfacial decohesion on the piezoresistive properties of graphene/polymer nanocomposite. A cohesive zone model is identified by atomistic simulations. This cohesive zone model enriches imperfect interfaces, which model graphene sheets, at mesoscale in our continuum mechanical model. This nonlinear mechanical model is used to generate deformed representative volume element to study influence of strain and interfacial decohesion on the conductivity of graphene/polymer nanocomposites. The effective conductivity is studied with an electric continuum model at mesoscale that incorporates the tunneling effect. A conductor-insulator transition is observed for elongations above $2 \%$ for graphene volume fraction just above the percolation threshold. The transition appears for an elongation of $8 \%$ instead of $2 \%$, when the interfacial decohesion is removed.
\end{abstract}

Keywords: polymer graphene nanocomposites, imperfect interface, cohesive zone model, electromechanical coupling

Submitted to: Modelling Simul. Mater. Sci. Eng.

\section{Introduction}

Graphene/polymer nanocomposites have recently attracted a growing attention due to their high electric conductivity for very low volume fraction [1-7] and their interesting mechanical performances [4-12]. A wide range of smart materials have been developed for practical applications with the introduction of graphene or other carbon allotropes [13-15]. In particular, the research on strain sensing behavior of graphene/polymer nanocomposites has been carried out based on monitoring the strain-induced resistivity 
change, i.e. piezoresistive effect [16-24], showing potential in the area of structural health monitoring.

Although many experiments have been conducted to study these new materials, the mechanisms underlying the piezoresistive effect are still not well understood. Their influences on effective properties remain an open domain. This paper proposes a multiscale and mutli-physical strategy, in order to understand the role of interfacial decohesion on the piezoresistive properties of graphene/polymer nanocomposite. The main issues of this aim are:

- the identification of the mechanical behavior laws associated with the nanometric decohesion mechanism between graphene and the polymer;

- the numerical simulation of Representative Volume Element (RVE) containing very thin objects such as graphene sheets;

- the modeling of quantum effects, such as the tunneling effect, at the continuum mesoscale.

In that context, a multiscale and multiphysics simulation framework, from nanoscale up to the macroscale, can help us to tackle these issues. The contribution and the originality of this work is to combine and to transpose three modeling framework :

- the identification by Molecular Dynamics (MD) of a Cohesice Zone (CZ) model between graphene and polymer [25];

- the imperfect interfaces to model the graphene sheets like a $2 \mathrm{D}$ object to avoid to finer mesh for the resolution of the mesoscopic problem by Finite Element Method (FEM) $[26,27]$;

- the introduction, at the continuum mesoscoale, of the tunneling effect to model of electrical conductivity through a distance function, $d(x)[26,27]$.

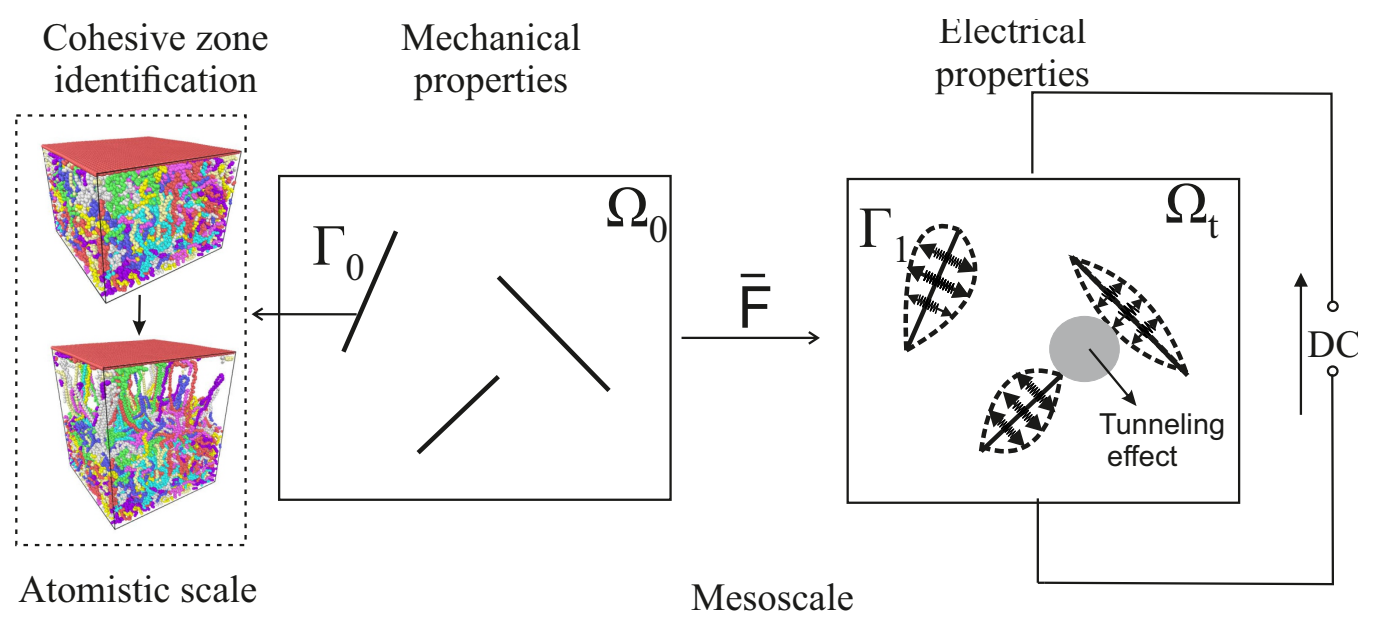

Figure 1. Strategy of the multiscale modeling for study the electromechanical coupling of graphene/polymer nanocomposites. 
Figure 1 presents a scheme of our bottom-up approach, which is able to predicts the variation of electrical conductivity and percolation threshold of the polymer/graphene nanocomposites under applied strain. The effective electrical conductivity is computed by nonlinear FEM framework, which takes into account the tunneling effect $[26,27]$, at mesoscale on the deformed RVEs. The tunneling effect, which is quantum phenomena, is created through very thin isolating barriers like polymer layers when the distance between the two conducting phases lower than several nanometers [28-30]. It leads to unexpected values of electrical conductivity for very small volume fractions of graphene [1-7]. The electrical percolation induced by tunneling effects have been also studied by discrete models of resistance networks [31-33] and by mean field theory [34-38]. However, the breaking of the percolating path induced by interfacial decohesion is a local phenomenon. Moreover, the desire to couple conductivity with mechanics has led us to take a full field continuum model [26, 27,39].

These RVEs are obtained by a nonlinear FEM mechanical framework, which deforms a random microstructure with imperfect interfaces [40-51]. The imperfect interfaces can be seen like a surface elasticity membrane [52-62] combine with CZ model [63-65]. The theory with elastic interface, called Gurtin-Murdoch theory, has been amply studied to model the size effects that are inherent to the nano scale [66$69, ?-72, ?-77]$. The CZ model has been amply studied in the past to model fracture in materials [78-93] or damage nucleation and decohesion in heterogenous materials [94100]. Note that the decohesion mechanism observed in this study is very similar to the polymer crazing, which are been also studied by CZ model [101-104].

At the atomistic level, the crazing [105-108] and the decohesion between carbon filler and polymer matrix [109-113] have been investigated without using the CZ model. First of all, the atomistic simulations have been performed to evaluate the $\mathrm{CZ}$ model parameters in crystalline solids [114-120]. Then these methods have been transposed to the study of the decohesion between filler and matrix in polymer nanocompoistes $[25,121-126]$. On another side, the identification of elastic parameter associated to surface elasticity can be conducted by atomistic simulations [120,127-133].

The paper is organized as following: in section 2, we provide the details on the identification of $\mathrm{CZ}$ model by $\mathrm{MD}$ simulations, for the graphene/polymer interface. In section 3, the mechanical model with imperfect interfaces are described with the formalism of the finite elasticity, and the effective stiffness moduli of the nanocomposites are estimated. Finally, the electrical modeling incorporating the tunneling effect is presented, and the numerical examples of the piezoresistive effect are presented in section 4 . 


\section{Identification of a nonlinear cohesive model by molecular dynamics}

\subsection{Graphene/polymer atomistic model}

In the present section, a nonlinear CZ model for the interface between graphene and polymer is identified by MD simulations. For this purpose, we study a sample where a graphene sheet is placed on the top of PE slab (see Fig. 2 (a)). A coarse-grained model is used for PE macromolecules, which are represented by 500 beads of $-\mathrm{CH}_{2}-$ atom units. The system contains $80 \mathrm{PE}$ chains and 4860 carbon atoms in the graphene sheet. The system is periodical on $\mathrm{X}-\mathrm{Y}$ plane and non-periodical on $\mathrm{Z}$ direction. Dreiding potential [134] is employed in the simulation. For simplicity, we assume that the system is nonpolar, i.e. the electrostatic term in the nonbonded part of potential energy are neglected.

\subsection{Preparation of amorphous polymer}

The initial system is prepared from the self-avoiding random walk (see, e.g., [135] for RVEiew) combining the molecular dynamics relaxation steps as introduced by [136] and used by [137] to generate homopolymers and copolymers. The different steps of this algorithm are :

(i) Initialization : the atoms inside the graphene sheet are arranged on the top of the simulation box.

(ii) Chain nucleation : the first atom of each chain is randomly placed with a uniform probability density inside the box deprived of a layer of thickness of $2 \AA$ from the graphene sheet.

(iii) Chain growth : all chains grow at each step of the random walk with the random order. The degrees of freedom associated with the hardest term of the valence or bond part of potential energy are maintained at their equilibrium position, i.e. the distance between two atom units and the bending angle between two bonds are fixed. The possible position of the next atom unit during the random walk is on a circle under these geometric constraints. The set of $N_{t}$ trial positions $\left\{\mathbf{r}_{\alpha+1}\right\}=\left\{\mathbf{r}_{\alpha+1}^{(1)}, \ldots, \mathbf{r}_{\alpha+1}^{\left(N_{t}\right)}\right\}$ is chosen with uniform distribution on this circle. The potential energy, $\mathcal{U}\left(\mathbf{r}_{1}, \ldots, \mathbf{r}_{\alpha}, \mathbf{r}_{\alpha+1}^{(i)}\right)$, for each trial position is computed. The position is randomly chosen with a probability distribution according to the Boltzmann weight

$$
p\left(\mathbf{r}_{\alpha+1}^{(i)}\right) \propto \exp \left(-\frac{\mathcal{U}\left(\mathbf{r}_{1}, \ldots, \mathbf{r}_{\alpha}, \mathbf{r}_{\alpha+1}^{(i)}\right)}{k_{b} T}\right)
$$

where $k_{b}$ is the Boltzmann constant and the temperature $T=300 \mathrm{~K}$. It interesting to note that the chains can grow from both extremities.

(iv) Chain relaxation : an annealing is performed by a molecular dynamics simulation with Berendsen thermostat [138] at the temperature of $700 \mathrm{~K}$ during 
20 ps. The position of atoms in graphene sheet are frozen at the same position. This relaxation step is performed when the chains are 50, 100, 150, 200, 250, 300, 350, 400, 450, 500 monomers. The algorithm come back to step (iii) at the end of relaxation until there are 500 atom units per chain.

(v) Final relaxations and quenching : after the chain growth, the system is then first relaxed at NpT ensemble via Nosé-Hoover $[139,140]$ at $\mathrm{T}=700 \mathrm{~K}$ and $\mathrm{p}=0$ atm for 10000 fs and followed by a cooling from $700 \mathrm{~K}$ to $200 \mathrm{~K}$ during 40000 fs. Note that for all the pRVEious steps, the system is periodic, i.e. there are two graphene polymer interfaces. We increase the distance of $10 \mathrm{~nm}$ between the upper side of graphene and the polymer to keep only one interface. Then, a second $\mathrm{NpT}$ relaxation at $\mathrm{T}=200 \mathrm{~K}$ and $\mathrm{p}=0 \mathrm{~atm}$ for $20000 \mathrm{fs}$. Another relaxation during $20000 \mathrm{fs}$ is performed at NVE ensemble. The last step is the quenching at $0 \mathrm{~K}$ by a minimization of the system potential energy. During these last three steps, we ensure that the distance between the upper side of graphene and the polymer always remains greater than 2 times the global cutoff of interatomic potentials.

\subsection{Identification of cohesive zone model}

To study the separation in opening mode, graphene was moved in successive steps of $0.5 \AA$ along the $\mathrm{Z}$ direction following by a minimization procedure. The graphene atoms and bottom layer of the polymer were kept fixed (see Fig. 2(a)). The separation process is depicted in Figs. 2. The polymer chains undergo stretch at the beginning along the $\mathrm{Z}$ direction, then form highly oriented structures, called fibrils or nano-fibrils. Voids appear between the fibrils during the decohesion. This deformation mechanism observed during the simulation is similar to the nano-crazes of some semi-crystalline polymers, such as polybutene $[141,142]$. The size of the void grows along the separation direction; and the extended chains slide along the graphene sheet to increase the fibrils as described in the reference RVEiew paper of Kramer and Berger [143]. It should be noted that the separation is controlled by the chain desorption at the graphene surface by sliding, which is dominated by van der Walls interaction.

The average force of polymer on graphene was monitored, as from which we can get the normal traction force, $t_{n}$ of cohesive zone as a function of the displacement of graphene layer, $\llbracket u_{n} \rrbracket$, as shown in Fig. 3. The force varies linearly with the displacement of graphene sheet at the which corresponds to the domain where the behavior of the interface is RVEersible. Then the curve bends to reach a maximum at $0.7 \mathrm{~nm}$, called the yield threshold. This phase corresponds to the nano-fribils creation and to the cavity initiation. Once the yield threshold crosses, the force decreases with the displacement of graphene sheet. During this phase, the chains slip on the graphene sheet to feed the fibrils. It is likely that the observed softening is related to the reduction of the contact area between polymer chains and graphene. The MD results are fitted with the 


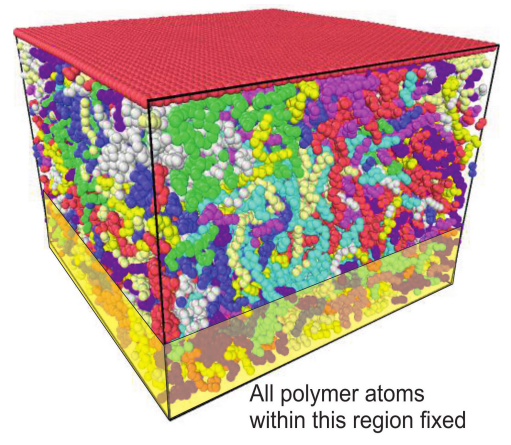

(a)

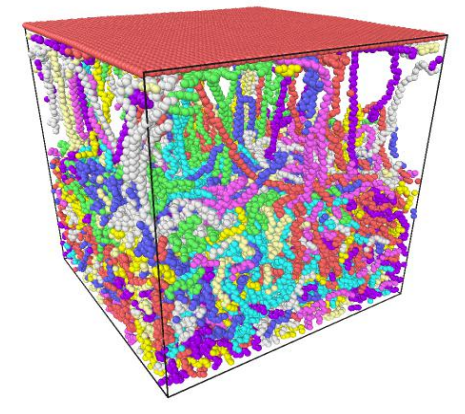

(c)

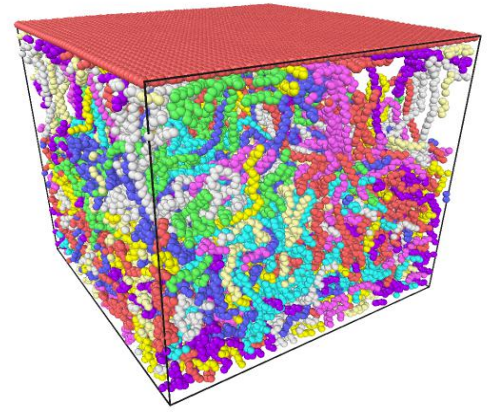

(b)

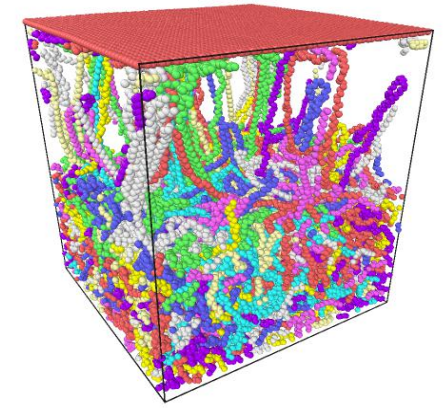

(d)

Figure 2. Evolution of the atomistic model during the normal separation. The model contains 44860 atoms, and the graphene is moved with a step of $0.5 \AA$. The graphene atoms and the bottom layer of the polymer are fixed during the relaxation.

following empirical model:

$$
t_{n}=g_{c z}\left(\llbracket u_{n} \rrbracket\right)= \begin{cases}-1529 \llbracket u_{n} \rrbracket^{2}+2150 \llbracket u_{n} \rrbracket & \text { if } 0 \leq \llbracket u_{n} \rrbracket<0.7 \\ \frac{65}{\llbracket u_{n} \rrbracket^{8}}-\frac{4.31}{\llbracket u_{n} \rrbracket^{14}}+263.74 & \text { if } 0.7 \leq \llbracket u_{n} \rrbracket \leq 1.15 \\ 360 \exp \left(-0.16 \llbracket u_{n} \rrbracket\right)-15.12 & \text { if } \llbracket u_{n} \rrbracket>1.15\end{cases}
$$

\section{Mechanical modeling}

In this section, we use the theory of nonlinear continuum mechanics which details can be found in [144-147] among others. Consider a continuum body $\Omega$ in the reference configuration $\Omega_{0} \in \mathbb{R}^{3}$, and the spatial configuration $\Omega_{t} \in \mathbb{R}^{3}$. The boundary of $\Omega_{0}$ are denoted by $\partial \Omega_{0}$ in reference configurance and $\partial \Omega_{t}$ in actual configuration. In the reference configuration, the graphene sheets are distributed randomly in the domain as the internal discontinuity $\Gamma_{0}^{(n)}(n=1,2, \ldots, N)$, as shown in Fig. 4. The graphene surfaces and their boundary are collectively denoted by $\Gamma_{0}=\cup_{n} \Gamma_{0}^{(n)}$ and $\partial \Gamma_{0}=\cup_{n} \partial \Gamma_{0}^{(i n)} \cup \partial \Gamma_{0}^{(o u t)}$ where $\partial \Gamma_{0}^{(i n)}=\partial \Omega_{0} \cap \partial \Gamma_{0}$. The two sides of the interface are denoted by $\Gamma_{0}^{+}$and $\Gamma_{0}^{-}$. And the unit vector normal to the interface in the reference configuration is $\mathbf{n}(\mathbf{X})$. The displacement of the bulk, and the two sides of the interface are $\mathbf{u}, \mathbf{u}^{-}$and $\mathbf{u}^{+}$respectively. The current positions, $\mathbf{x}$ of the material particles at the 


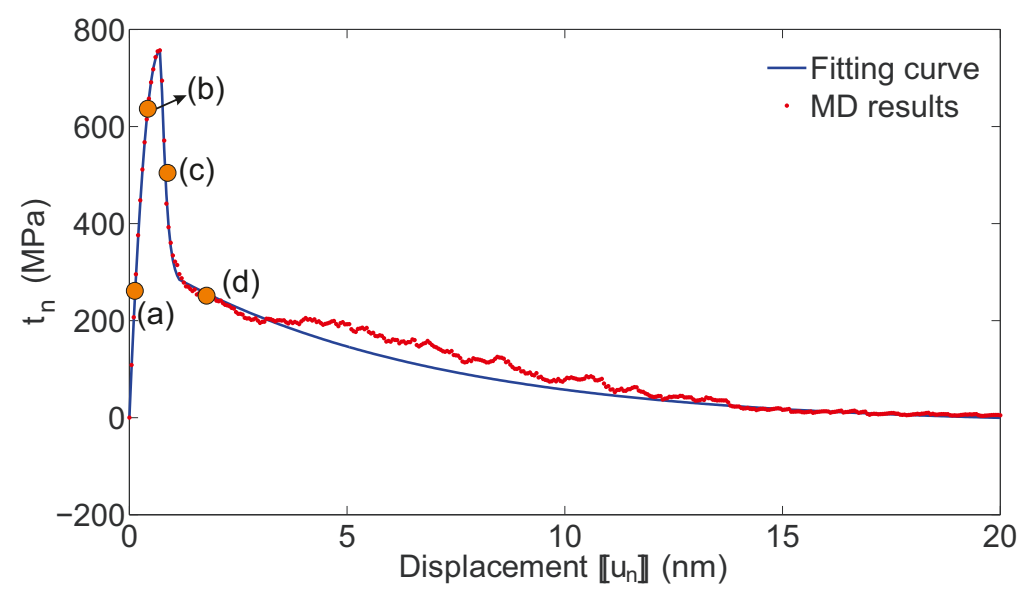

Figure 3. Traction force, $t_{n}$ vs displacement of graphen layer $\llbracket u_{n} \rrbracket$. The points denote the MD results and the line is the fitting curve. There is a correspondence between the bold points with the letters (a-d) on the curve and the Figs. 2(a-d).
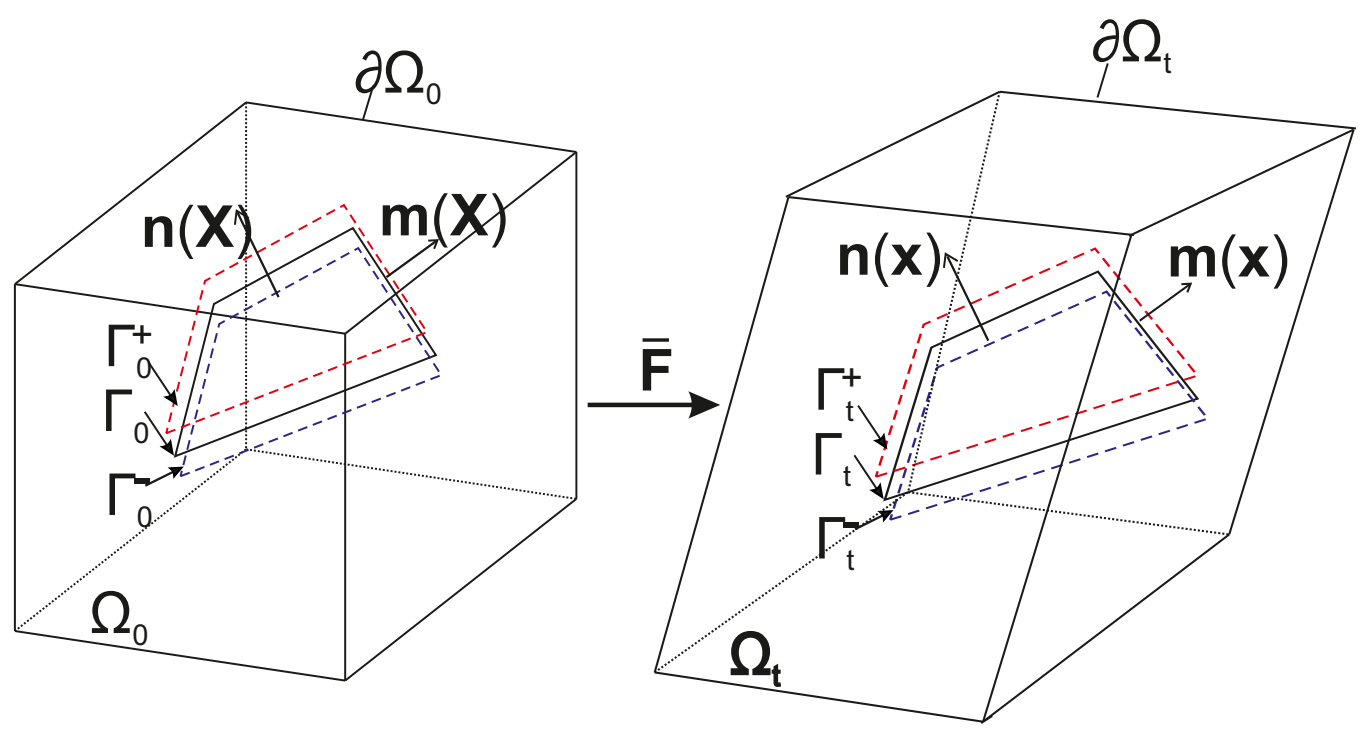

Figure 4. RVE model of the graphene-reinforced nanocomposite.

inital position, $\mathbf{X}$, are defined by $\mathbf{x}=\mathbf{X}+\mathbf{u}$ for the bulk. $\mathbf{x}^{-}$and $\mathbf{x}^{+}$are the current position for the two sides of interface. The graphene sheets are modeled as the general imperfect interface $[49,50]$, satisfying $\llbracket \mathbf{u} \rrbracket=\mathbf{u}^{+}-\mathbf{u}^{-} \neq 0$ and $\llbracket \mathbf{x} \rrbracket \neq 0$, the traction through the graphene surfaces is also discontinous, $\llbracket \mathbf{t} \rrbracket=\mathbf{t}^{+}-\mathbf{t}^{-} \neq 0$ where $\mathbf{t}^{+}$and $\mathbf{t}^{-}$ are respectively the traction on $\Gamma_{0}^{+}$and $\Gamma_{0}^{-}$associated to the normal $\mathbf{n}(\mathbf{X})$.

\subsection{Internal virtual work}

In this section we follow the theory of imperfect interface at finite strains developed by Javili et al [50]. The internal virtual work, $\delta W_{\text {int }}$, is given in reference configuration by the contributions of polymer bulk and graphene sheets, which are modeled by imperfect 
interfaces:

$$
\delta W_{\text {int }}(\mathbf{u}, \delta \mathbf{u})=\delta W_{\text {int }}^{(b)}(\mathbf{u}, \delta \mathbf{u})+\delta W_{\text {int }}^{(s)}(\mathbf{u}, \delta \mathbf{u})
$$

The polymer bulk contribution is

$$
\delta W_{\text {int }}^{(b)}(\mathbf{u}, \delta \mathbf{u})=\int_{\Omega_{0}} \mathbf{P}: \delta \mathbf{F} \mathrm{d} V
$$

where $\mathbf{P}$ is the first Piola-Kirchoff stress and $\delta \mathbf{F}=\nabla_{X} \delta \mathbf{u}$ is the gradient of the virtual displacement $\delta \mathbf{u}$ with respect to the Lagrangian coordinate, $\mathbf{X}$.

The contribution of imperfect interfaces is given by

$$
\delta W_{\text {int }}^{(s)}(\mathbf{u}, \delta \mathbf{u})=\int_{\Gamma_{0}} \mathbf{P}^{(\mathbf{s})}: \delta \mathbf{F}^{(s)}+\{\{\mathbf{t}\}\} \cdot \llbracket \delta \mathbf{u} \rrbracket \mathrm{d} S
$$

where $\mathbf{P}^{(\mathbf{s})}$ is surface first Piola-Kirchof stress and $\delta \mathbf{F}^{(s)}=\delta \mathbf{F} . \mathbf{I}_{\mathbf{0}}^{\mathbf{s}}$, denotes the surface gradient of the virtual displacement, where $\mathbf{I}_{\mathbf{0}}^{\mathbf{s}}=\mathbf{I}-\mathbf{n}(\mathbf{X}) \otimes \mathbf{n}(\mathbf{X})$ is the projector into the tangente plan of interface in the reference configuration. $\{\{\mathbf{t}\}\}=1 / 2\left(\mathbf{t}^{+}+\mathbf{t}^{-}\right)$is the average of traction. Note that the expression of internal virtual work with imperfect interfaces is true assuming that the interface motion to the midplane [50].

Using the second Piola-Kirchhoff stress tensor $\mathbf{S}=\mathbf{F}^{-1} \mathbf{P}$ (resp. the surface second Piola-Kirchhoff stress tensor, $\mathbf{S}^{s}=\left(\mathbf{F}^{s}\right)^{-1} \mathbf{P}^{s}$ ), we achieve finally the following expression of internal virtual work (for a configuration in static equilibrium) in the form

$$
\begin{aligned}
& \delta W_{i n t}^{(b)}(\mathbf{u}, \delta \mathbf{u})=\int_{\Omega_{0}} \mathbf{S}: \delta \boldsymbol{\varepsilon} \mathrm{d} V \\
& \delta W_{\text {int }}^{(s)}(\mathbf{u}, \delta \mathbf{u})=\int_{\Gamma_{0}} \mathbf{S}^{\mathbf{s}}: \delta \boldsymbol{\varepsilon}^{\boldsymbol{s}}+\{\{\mathbf{t}\}\} \cdot \llbracket \delta \mathbf{u} \rrbracket \mathrm{d} S
\end{aligned}
$$

where $\delta \boldsymbol{\varepsilon}\left(\right.$ resp. $\left.\delta \varepsilon^{s}\right)$ is the variation of the symmetric Green-Lagrange strain tensor, $\varepsilon=\frac{1}{2}\left(\mathbf{F}^{T} \mathbf{F}-\mathbf{I}\right)$ (resp. the variation of the surface Green-Lagrande strain tensor, $\left.\varepsilon^{s}=\mathbf{I}_{\mathbf{0}}^{\mathbf{s}} \varepsilon \mathbf{I}_{\mathbf{0}}^{\mathbf{s}}\right)$.

\subsection{Constitutive laws}

We choose the Saint Venant-Kirchhoff model for bulk part, which is an extension of the linear elastic material model, the second Piola-Kirchoff stress is given by :

$$
\mathbf{S}=\mathbb{C}^{(b)}: \varepsilon
$$

where $\mathbb{C}^{(b)}$ is the forth order tensor of stiffness. It is assume to be isotropic and defined by Lame's coefficient $\lambda^{(b)}=6890 \mathrm{MPa}$ and $\mu^{(b)}=680 \mathrm{MPa}$ [26]. These elastic parameters are identified by deformation of MD simulation box of pur PE following the classical procedure [148].

Like for the bulk, we assume for sake of simplicity that the behavior of imperfect interface is RVEersible. The surface elastic behavior of graphene is assumed to isotropic 
inside its plane, so the surface second Piola-Kirchoff stress is given also by Saint VenantKirchhoff model :

$$
\mathbf{S}^{\mathbf{s}}=2 \mu^{(s)} \boldsymbol{\varepsilon}^{\boldsymbol{s}}+\lambda^{(s)}\left(\boldsymbol{\varepsilon}^{\boldsymbol{s}}: \mathbf{I}_{\mathbf{0}}^{\mathbf{s}}\right) \mathbf{I}_{\mathbf{0}}^{\mathbf{s}}
$$

where the surface Lame's coefficient $\lambda^{(s)}=19.0 \mathrm{~N} . \mathrm{m}^{-1}$ and $\mu^{(s)}=18.7 \mathrm{~N} . \mathrm{m}^{-1}$ are identified by MD simulation [149].

The expression of traction $\{\{\mathbf{t}\}\}$ is assume to be aligned with the displacement jump $\llbracket \mathbf{u} \rrbracket$ and given by :

$$
\{\{\mathbf{t}\}\}=g_{c z}(\llbracket \mathbf{u} \rrbracket) \frac{\llbracket \mathbf{u} \rrbracket}{\|\llbracket \mathbf{u} \rrbracket\|}
$$

where $g_{c z}(\llbracket \mathbf{u} \rrbracket)$ is the function identified by MD simulations in Eq. 2.

\subsection{Weak form of mechanical problem}

In order to relate the micro stress and strain feilds to the imposed macroscopic strain, the effective quantities are defined as [50],

$$
\overline{\mathbf{F}}=\frac{1}{\left\|\Omega_{0}\right\|} \int_{\Omega_{0}} \mathbf{F} \mathrm{d} V+\frac{1}{\left\|\Omega_{0}\right\|} \int_{\Gamma_{0}} \llbracket \mathbf{u} \rrbracket \otimes \mathbf{n}(\mathbf{X}) \mathrm{d} S
$$

and

$$
\overline{\mathbf{P}}=\frac{1}{\left\|\Omega_{0}\right\|} \int_{\Omega_{0}} \mathbf{P} \mathrm{d} V+\frac{1}{\left\|\Omega_{0}\right\|} \int_{\Gamma_{0}} \mathbf{P}^{\mathbf{s}} \mathrm{d} S
$$

where $\overline{\mathbf{F}}$ and $\overline{\mathbf{P}}$ are the effective deformation gradient and effective first Piola-Kirchoff stress respectively. The effective Green-Lagrange strain tensor $\bar{\varepsilon}$ and the effective second Piola-Kirchoff stress $\overline{\mathbf{S}}$, are defined by :

$$
\bar{\varepsilon}=\frac{1}{2}\left(\overline{\mathbf{F}}^{T} \overline{\mathbf{F}}-\mathbf{I}\right) \quad \text { and } \quad \overline{\mathbf{S}}=\overline{\mathbf{F}}^{-1} \overline{\mathbf{P}}
$$

To impose that the incremental internal virtual work at micro scale, $\delta W_{\text {int }}$, is egal to the macroscopic internal virtual work, $\overline{\delta W}_{i n t}=\overline{\mathbf{P}} \delta \overline{\mathbf{F}}$, we chose periodic boundary conditions to statisfy the extended Hill-Mandel condition [50]. The weak form associated to mechanical problem at micro scale is :

Find $\mathbf{u} \in H^{1}\left(\Omega_{0}\right)$, satisfying the boundary conditions $\mathbf{u}=\overline{\mathbf{F}} \cdot \mathbf{x}+\tilde{\mathbf{u}}$ over $\partial \Omega_{0}$ with $\tilde{\mathbf{u}}$ periodic, such as

$$
\int_{\Omega_{0}} \mathbf{S}(\mathbf{u}): \delta \boldsymbol{\varepsilon}(\mathbf{u}, \delta \mathbf{u}) \mathrm{d} V+\int_{\Gamma_{0}} \mathbf{S}^{\mathbf{s}}(\mathbf{u}): \delta \boldsymbol{\varepsilon}^{\boldsymbol{s}}(\mathbf{u}, \delta \mathbf{u})+\{\{\mathbf{t}(\llbracket \mathbf{u} \rrbracket)\}\} \cdot \llbracket \delta \mathbf{u} \rrbracket \mathrm{d} S=0(14)
$$

forall $\delta \mathbf{u} \in H_{0}^{1}\left(\Omega_{0}\right)$.

We use FEM to discretize the solution space, linear tetrahedrons for bulk part and linear triangles for interfaces. A Newton-Raphson procedure is used to solve this non-linear problem step by step for small increment, $\Delta \overline{\mathbf{F}}$, of effective strain gradient. 


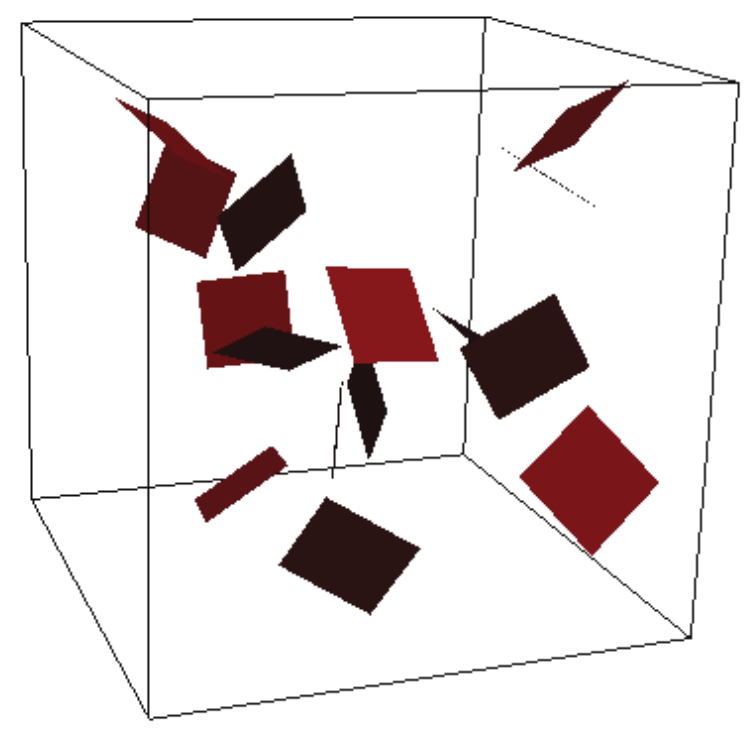

Figure 5. RVE for the graphene/polymer nanocomposites involving 15 graphene square sheets in a cube of $70 \times 70 \times 70 \mathrm{~nm}^{3}$.

\subsection{Effectives mechanical properties}

We use this FEM framework to investigate the effective mechanical properties of graphene reinforced nanocomposites with various graphene volume fraction in linear regime. The volume fraction is controled by increasing the number of graphene sheets in the domain. The generation of the random RVEs is provided in appendix Appendix A. The in-plane dimensions of graphene sheets are $15 \times 15 \mathrm{~nm}^{2}$ and the RVE side length of the cubic domain is $70 \mathrm{~nm}$. We consider multi-layer graphene platelets, also called here sheets, which have a finite thickness, $h=0.2 \mathrm{~nm}$. In this study, the graphene sheet are modeled by square plane with the side length $L=15 \mathrm{~nm}$. This assumption is due to extremely thin thickness of graphene and relies on the imperfect surface model. One example of isotropic graphene nanocomposite RVE is illustrated in Fig. 5.

The numerical results are provided in Fig. 6 for graphene/PE nanocomposite with varying graphene volume fractions. With randomly distributed graphene, the samples present isotropic properties. The effective Young's modulus $E$ and effective shear modulus $G$ are plotted for each volume fraction as shown in Fig.6, where the average values are obtained for 10 realizations. It indicates that the elastic moduli of the graphene-reinforced nanocomposites increases with the graphene volume fraction. As expected, as compared with the computational results without the $\mathrm{CZ}$ model, the introduction of interface decreases the stiffness of the composites, and playes an important role especially at large graphene volume fraction. An increase of around $30 \%$ of the moduli is exhibited when the graphene volume fraction reaches 1.5 vol\%. The experimental results in some literatures $[8,29]$ show $50 \%$ and $62 \%$ improvement of Young's modulus by the addition of $1.5 \mathrm{wt} \%$ functionalized graphene sheets and 0.7 wt $\%$ graphene oxide respectively. There are several possible reasons 


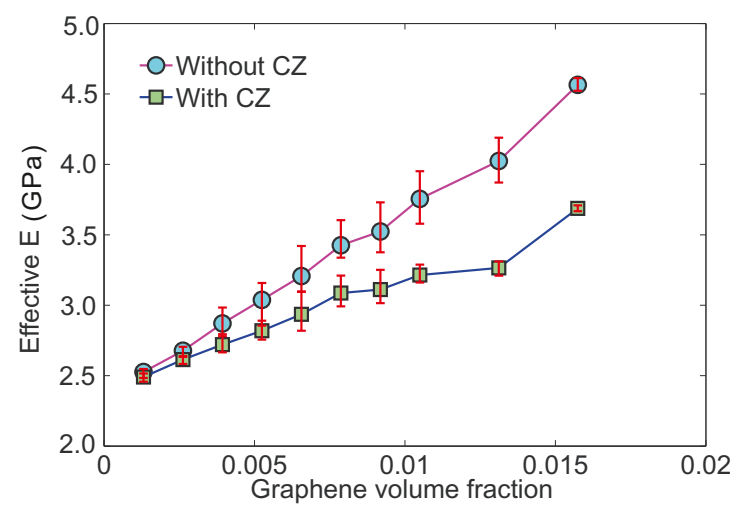

(a)

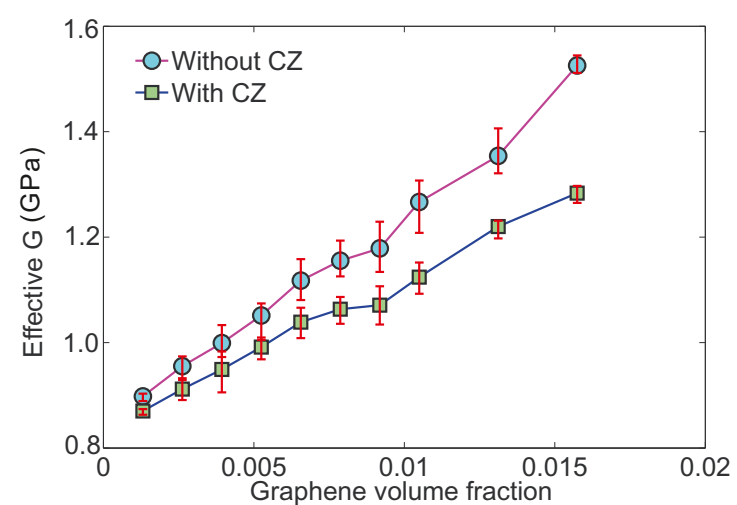

(b)

Figure 6. Effective Young's moduli and shear moduli along with the increase of graphene volume fraction. Graphene sheets are randomly distributed in the RVE, which has the side length of $70 \mathrm{~nm}$. The dimensions of graphene sheets are $15 \times 15 \times 0.2$ $\mathrm{nm}^{3}$.

for the higher experimental results compared to our numerical results: firtly, the aspect ratio of graphene in the experiments can be larger than our assumption in the numerical example; secondly, the functionalized graphene sheets and graphene oxide show enhancemenent of the interfacial interaction with the polymer matrix.

\section{Electro-mechanical coupling examples}

\subsection{Electrical model of graphene-reinforced composites}

A numerical model for electric properties of graphene/polymer nanocomposites has been proposed in [26]. In this section, we use the deformed RVE in actual conficuration $\Omega_{t}$ providing from mechanical simulations. The graphene sheets are assumed to be in the middle of imperfect interface $\Gamma_{t}$. According to the extremely thin thickness, the graphene sheets are modeled by highly conducting imperfect surfaces [150]. Electric tunneling effect between graphene sheets originating from the nanoscale is taken into account.

The electric power, $\mathcal{P}_{\text {elec }}$ of the system in actual configuration is defined by

$$
\mathcal{P}_{\text {elec }}=\int_{\Omega_{t}} \omega^{(b)}(\mathbf{x}) \mathrm{d} V+\int_{\Gamma_{t}} \omega^{(s)}(\mathbf{x}) \mathrm{d} S,
$$

where the density functions $\omega^{(b)}$ and $\omega^{(s)}$ are the bulk and surface density functions expressed by

$$
\omega^{(b)}(\mathbf{x})=\frac{1}{2} \mathbf{j}(\mathbf{x}) \cdot \mathbf{E}(\mathbf{x}) \text {, and } \quad \omega^{(s)}(\mathbf{x})=\frac{1}{2} \mathbf{j}^{\mathbf{s}}(\mathbf{x}) \cdot \mathbf{E}^{\mathbf{s}}(\mathbf{x}) .
$$

In these equations above, $\mathbf{E}(\mathbf{x})$ and $\mathbf{j}(\mathbf{x})$ denote to the electric field and current density respectively, and $\mathbf{E}(\mathbf{x})$ is related to the electric potential $\phi$ by $\mathbf{E}(\mathbf{x})=-\nabla_{x} \phi(\mathbf{x})$ where $\nabla_{x}$ is the gradient with respect to the Eulerian coordinate, $\mathbf{x}$. Besides, $\mathbf{E}^{\mathbf{s}}(\mathbf{x})$ and $\mathbf{j}^{\mathbf{s}}(\mathbf{x})$ 
are the surface electric field and surface current density with respect to the graphene sheets, where $\mathbf{E}^{\mathbf{s}}=\mathbf{I}_{\mathbf{t}}^{\mathbf{s}}$. $\mathbf{E}$ with $\mathbf{I}_{\mathbf{t}}^{\mathbf{s}}=\mathbf{I}-\mathbf{n}(\mathbf{x}) \otimes \mathbf{n}(\mathbf{x})$ the projector operator characterizing the projection of a vector along the tangent plane to $\Gamma_{t}$ at a point $\mathbf{x} \in \Gamma_{t}$ and $\mathbf{n}(\mathbf{x})$ is the unit normal vector to $\Gamma_{t}$ in actual configuration.

The local constitutive equations relating $\mathbf{j}$ and $\mathbf{E}$ are nonlinear as:

$$
\mathbf{j}= \begin{cases}\mathbf{K}_{\mathbf{0}}^{(\mathbf{p})} \mathbf{E} & \text { if } d(\mathbf{x})>d_{\text {cut }}, \\ \mathcal{G}(\mathbf{E}, d(\mathbf{x})) \frac{\mathbf{E}}{|\mathbf{E}|} & \text { if } d(\mathbf{x})<d_{\text {cut }}\end{cases}
$$

where $d_{c u t}$ is a cut-off distance above which the tunneling effect can be neglected, and $\mathbf{K}_{\mathbf{0}}^{(\mathbf{p})}$ is the second-order tensor of electric conductivity of the polymer when neglecting tunneling effect. The polymer matrix is assumed to have an isotropic conductivity, i.e. $\mathbf{K}_{\mathbf{0}}^{(\mathbf{p})}=k_{0}^{(p)} \mathbf{I}$. Note that the relatively high value of $k_{0}^{(p)}=10^{-10} \mathrm{~S}^{-\mathrm{m}^{-1}}$ for polymer is choosen to assure the convergence of FEM framework to due the very high contrast between the conductivity of graphene and polymer matrix. The field, $d(\mathbf{x})$, called the distance function, is defined as the sum of the two smallest distances between the position $\mathbf{x}$ and the two neighbouring graphene sheets (see Fig. 7),

$$
d(\mathbf{x})=\min _{\mathbf{x}^{\Gamma} \in \Gamma^{i}}\left\|\mathbf{x}-\mathbf{x}^{\Gamma}\right\|+\min _{\mathbf{x}^{\Gamma} \in \Gamma^{j}, j \neq i}\left\|\mathbf{x}-\mathbf{x}^{\Gamma}\right\| .
$$

This function is updated for all deformed configurations of RVE.

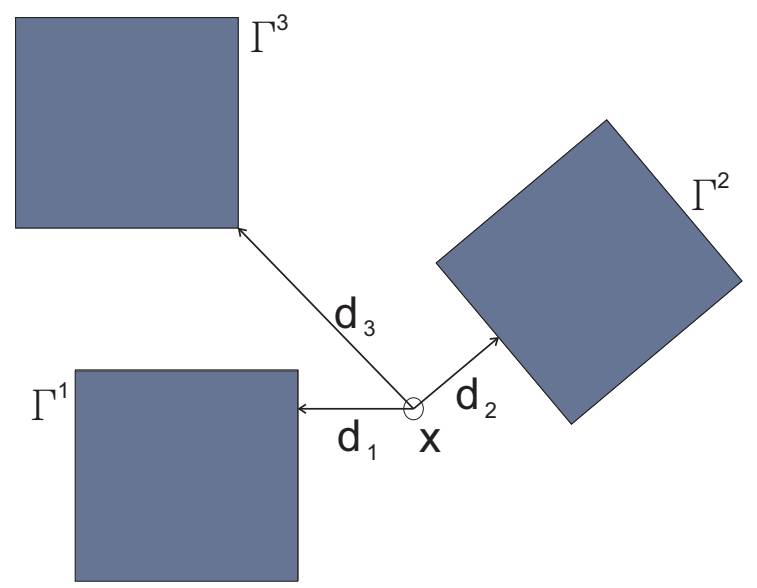

Figure 7. Distances of a point $\mathbf{x}$ from surrounding graphene sheets to compute the distance function, $d(\mathbf{x})$. Here, $d(\mathbf{x})=d_{1}+d_{2}$.

An explicit formula for the electric tunneling effect through a potential square barrier was first derived by Simmons [151] as:

$$
\begin{aligned}
\mathcal{G}(\mathbf{E}, d(\mathbf{x}))= & \frac{2.2 e^{3}}{8 \pi h_{p} \Phi_{0}}\|\mathbf{E}\|^{2} \exp \left[-\frac{8 \pi \Phi_{0} \sqrt{2 m \Phi_{0}}}{2.96 h_{p} e} \frac{1}{\|\mathbf{E}\|}\right] \ldots \\
& +\frac{3 e^{2} \sqrt{2 m \Phi_{0}}}{2 h_{p}^{2}}\|\mathbf{E}\| \exp \left[-\frac{4 \pi \sqrt{2 m \Phi_{0}}}{h_{p}} \frac{1}{d(\mathbf{x})}\right]
\end{aligned}
$$

where $\Phi_{0}$ is the energy barrier height that the electrons cross and $h_{p}, e$ and $m$ denote Plank's constant, the charge of an electron and a material parameter. 
The surface current density $\mathbf{j}^{\mathbf{s}}$ of the graphene sheet $\Gamma_{t}$ is related to the surface electric field, $\mathbf{E}^{\mathbf{s}}$ through (see e.g., $[47,150]$ ):

$$
\mathbf{j}^{\mathrm{s}}(\mathbf{x})=\mathbf{K}^{(\mathbf{s})} \mathbf{E}^{\mathrm{s}}
$$

where

$$
\mathbf{K}^{(\mathbf{s})}=h \mathbf{K}^{\star}, \quad \mathbf{K}^{\star}=\mathbf{K}^{(\mathrm{g})}-\frac{\left(\mathbf{K}^{(\mathrm{g})} \mathbf{n}(\mathbf{x})\right) \otimes\left(\mathbf{K}^{(\mathrm{g})} \mathbf{n}(\mathbf{x})\right)}{\mathbf{K}^{(\mathrm{g})}:(\mathbf{n}(\mathbf{x}) \otimes \mathbf{n}(\mathbf{x}))} .
$$

Here, $h$ is the thickness of graphene sheets and $\mathbf{K}^{(\mathbf{g})}$ denotes the second-order electric conductivity tensor of the bulk graphite, which is given by

$$
\mathbf{K}^{(\mathbf{g})}=k_{\|}^{(g)} \mathbf{I}_{\mathbf{t}}^{(\mathbf{s})}+k_{\perp}^{(g)} \mathbf{n}(\mathbf{x}) \otimes \mathbf{n}(\mathbf{x})
$$

where $k_{\|}^{(g)}=83200 \mathrm{~S} . \mathrm{m}^{-1}$ and $k_{\perp}^{(g)}=83.2 \mathrm{~S} . \mathrm{m}^{-1}$ are the conductivity parameter of graphen multi-layer from [1].

Considering the constitutive equations above, and minimizing the dissipated power with respect to the electric potential field, the weak form for electrical problem is : Find $\phi \in H^{1}\left(\Omega_{t}\right)$, satisfying the boundary conditions $\phi(\mathbf{x})=-\overline{\mathbf{E}} \cdot \mathbf{x}+\tilde{\phi}(\mathbf{x})$ over $\partial \Omega_{t}$ with $\tilde{\phi}(\mathbf{x})$ periodic, such as

$$
\int_{\Omega_{t}} \mathbf{j}(\phi) \cdot \nabla_{\mathbf{x}}(\delta \phi) \mathrm{d} V-\int_{\Gamma_{t}} \mathbf{j}^{(\mathbf{s})}(\phi) \cdot \mathbf{I}^{(\mathbf{s})} \nabla_{\mathbf{x}}(\delta \phi) \mathrm{d} S=0,
$$

forall $\delta \phi \in H_{0}^{1}\left(\Omega_{t}\right)$. Here $\overline{\mathbf{E}}$ is the effective electric field of the RVE.

We use a FEM to discretize the solution space, linear tetrahedrons for bulk part and linear triangles for graphene sheets. A Newton-Raphson procedure is used to solve this non-linear problem step by step at small increment, $\Delta \overline{\mathbf{E}}$, of effective electric field [26].

The problem being nonlinear, the effective conductivity is the incremental one dependent on the intensity and history of the applied electric field, which is defined as

$$
\left(\bar{K}_{T}\right)_{i j}(\overline{\mathbf{E}})=\frac{\partial \bar{j}_{i}(\overline{\mathbf{E}})}{\partial \bar{E}_{j}}
$$

where $\overline{\mathbf{j}}$ and $\overline{\mathbf{E}}$ denote the effective current density and effective electric field of the RVE respectively defined by :

$$
\overline{\mathbf{j}}=\frac{1}{\left\|\Omega_{t}\right\|} \int_{\Omega_{t}} \mathbf{j} \mathrm{d} V+\frac{1}{\left\|\Omega_{t}\right\|} \int_{\Gamma_{t}} \mathbf{j}^{(\mathbf{s})} \mathrm{d} S, \quad \overline{\mathbf{E}}=\frac{1}{\left\|\Omega_{t}\right\|} \int_{\Omega_{t}} \mathbf{E} \mathrm{d} V .
$$

\subsection{Electrical percolation threshold}

First, we investigate the influence of graphene volume fraction on the effective electric conductivity of graphene/polymer nanocomposites in the initial configuration $\Omega_{0}$. The barrier height between graphene and polymer matrix is taken as $\Phi_{0}=0.17 \mathrm{eV}$. The examples are given for a fixed value of effective electric field norm, $\|\overline{\mathbf{E}}\|=2.5 \mathrm{~V} \cdot \mu \mathrm{m}^{-1}$.

The numerical results are provided in Fig. 8 for graphene reinforced nanocomposite with varying graphene volume fraction. Taking into account the tunnel effect, the 


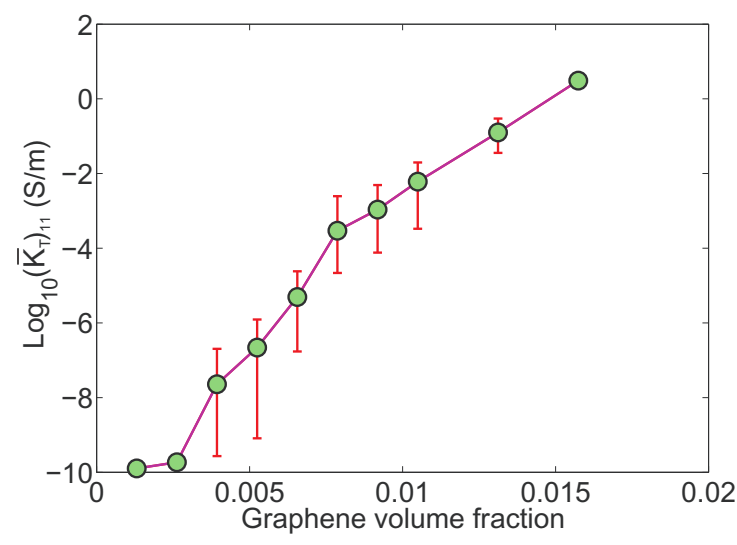

(a)

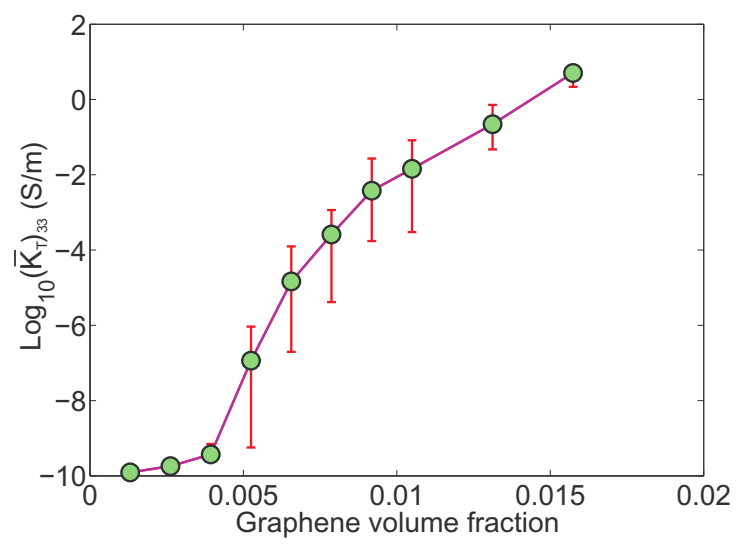

(c)

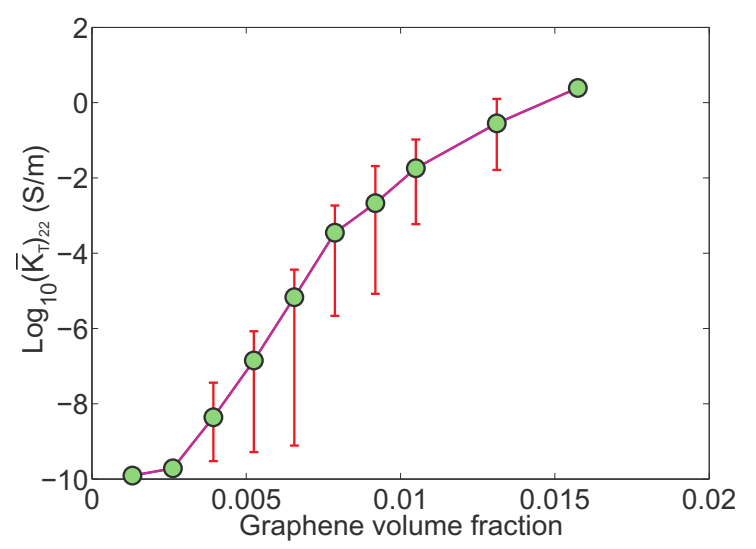

(b)

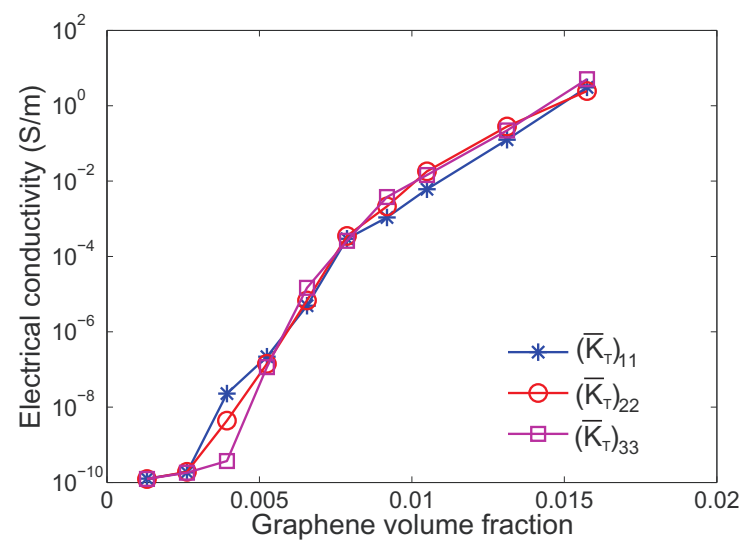

(d)

Figure 8. Effective electrical conductivity of graphene reinforced nanocomposites as a function of graphene volume fraction. Barrier height between graphene and polymer matrix is set to be $0.17 \mathrm{eV}$. The applied electric field is $0.0025 \mathrm{~V} / \mathrm{nm}$. (a) $\left(\bar{K}_{T}\right)_{11}$; (b) $\left(\bar{K}_{T}\right)_{22} ;(\mathrm{c})\left(\bar{K}_{T}\right)_{33} ;$ (d) Comparison.

numerical values of $\left(\bar{K}_{T}\right)_{11},\left(\bar{K}_{T}\right)_{22}$ and $\left(\bar{K}_{T}\right)_{33}$ are plotted for each volume fraction as shown in Fig. 8 (a-c), where the average values are obtained for 30 realizations. An additional comparison among the electrical conductivities in the three directions is shown in Fig. 8 (d). A sharp rise of conductivity of several orders of magnitude can be noticed at about 0.52 vol\% where the mean value exceeds $10^{-8} \mathrm{~S} / \mathrm{m}$. Generally, the percolation threshold is the minimum filler content in the matrix which is characterized by a sharp rise of several magnitude in conductivity due to the formation of conductive network, and realizes a transition from insulator to conductor. Therefore, we estimate that the percolation threshold is about $f_{c}=0.52 \mathrm{vol} \%$ in this example. The influence of barrier height, thickness of graphene multi-layer and their alignment have been investigated in [27]. 


\subsection{Evolution of electrical properties under stretching of the composite}

In this section, we study the impact of both streching and decohesion at the graphene polymer interface on the electric conductivity. Indeed, the conductivity is controled by tunneling effect, that depends strongly of ditance between graphene. Thefore, we impose an macroscopic elongation, $\bar{\varepsilon}=\bar{\varepsilon}_{11} \mathbf{e}_{1} \otimes \mathbf{e}_{1}$, on the RVEs, from $\bar{\varepsilon}_{11}=0 \%$ to $10 \%$. Due to the long comptutational time, only one RVE microstructure is randomly studied for each graphene volume fraction.

The deformed microstructures are stored for each $1 \%$ increment of deformation and the distance function, $d(\mathbf{x})$, is updated. Introducing the new distance function, the electrical conductivities at different effective strain $\bar{\varepsilon}_{11}$ are shown in Fig. 9. The boundary between insulator and condutor is defined to be $10^{-8} \mathrm{~S} / \mathrm{m}$, below which the material is supposed to be insulator. On the contrary, it is conductor. Focusing on the electrical conductivity along the direction of deformation $\left(\bar{K}_{T}\right)_{11}$, we can observe in Fig. 9 (a) that the mechanical deformation has little effect on the electrical conductivity of the nanocomposites when the graphene volume fraction is below the percolation threshold, $f<f_{c}=0.52 \mathrm{vol} \%$. When the graphene volume fraction is above the percolation threshold $\left(f>f_{c}=0.52 \mathrm{vol} \%\right)$, the electrical conductivity $\left(\bar{K}_{T}\right)_{11}$ decreases with the applied elongation, but it should be noted that the nanocomposites remains conductor. However, if the graphene volume fraction is around the percolation threshold ( $f \approx f_{c}=0.52$ vol\%), a sharp decrease of the electrical conductivity can be seen when the nanocomposites is subjected to strain, which is regarded as a transition point from conductor to insulator. For instance, with 0.66 vol\% graphene the transition point of the sample is $\bar{\varepsilon}_{11} \approx 3 \%$, and with $f=f_{c}=0.52 \operatorname{vol} \%$ graphene it is $\bar{\varepsilon}_{11} \approx 10 \%$. However, the elongation would not affect the electrical conductivities of the nanocomposites in the transverse directions $\left(\bar{K}_{T}\right)_{22}$ and $\left(\bar{K}_{T}\right)_{33}$, which can be seen from Fig. 9 (b-c). We note that this drop in conductivity does not occur for each curve above a volume fraction $f \geq$ $0.79 \mathrm{vol} \%$. This is probaly a consequence that the simulations for each volume fraction have been performed on only one realization, and that some configurations might be more favorable to this effect.

Observed this typical conductor-to-insulator transition for the composite with $f=0.66$ vol\% graphene by the proposed model, we compare the effective conductivity with the results which are estimated without considering the the decohesion between graphene and polymer matrix (i.e. we impose $\llbracket \mathbf{u} \rrbracket=0$ ). It can be seen on Fig. 10 that neglecting the cohesive interface, the transition point increases from $\bar{\varepsilon}_{11} \approx 3 \%$ to $8 \%$, which shows the important role of decohesion at the interface in predicting the piezoresisitivity properties of polymer graphene nanocomposite. It is interesting to note that it is theoretically possible to design a composite which can go from conductor to insulator by varying the applied strain on the system. This transition can be induced mainly by the decohesion for weak interfaces, or only by strain for a stronger interface but for a more important applied elongation.

Moreover, the influence of barrier height, $\Phi_{0}$, between graphene and polymer is 


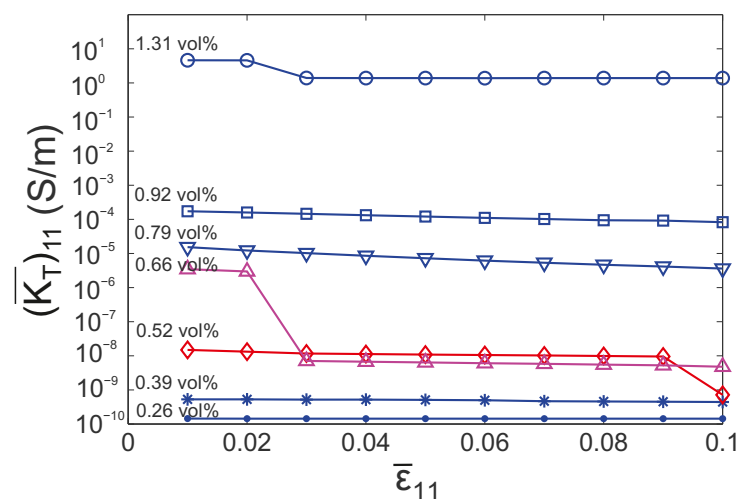

(a)

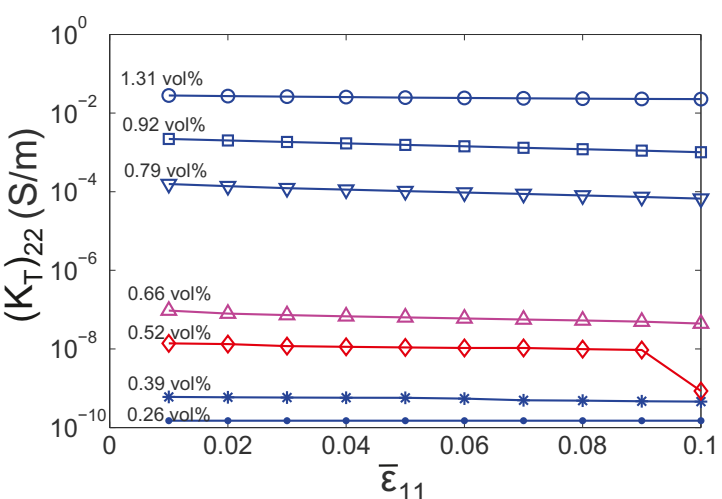

(b)

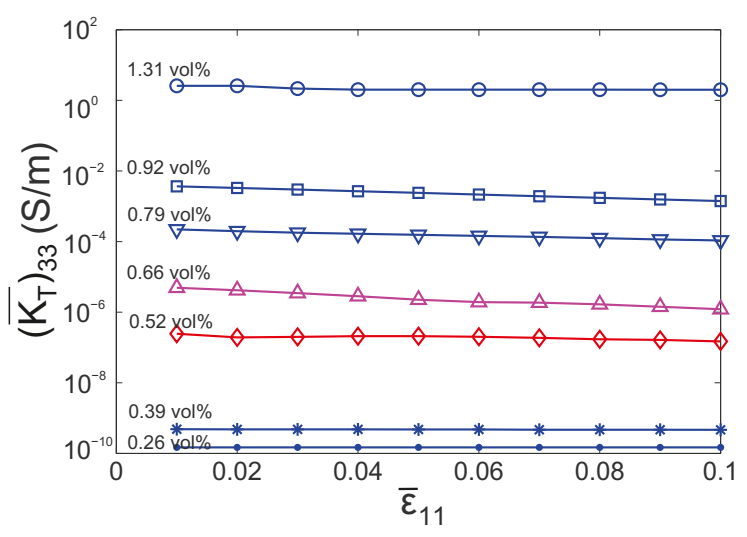

(c)

Figure 9. Effective electrical conductivity of graphene reinforced nanocomposites as a function of the deformation for various graphene volume fraction. Barrier height between graphene and polymer matrix is set to be $0.17 \mathrm{eV}$, and graphene aspect ratio is 75 . The applied electric field is $0.0025 \mathrm{~V} / \mathrm{nm}$. (a) $\left(\bar{K}_{T}\right)_{11} ;$ (b) $\left(\bar{K}_{T}\right)_{22} ;$ (c) $\left(\bar{K}_{T}\right)_{33}$.

presented in Fig. 11 for the configuration with 0.66 vol\% graphene sheets, which is just above the percolation threshold, $f_{c}$, and exhibits the conductor-to-insulator transition. It can be noted that effective conductivity of the composites decreases with the increasing barrier height, because the tunneling current goes down along the growing barrier height according to Eq. 20 and results in [27]. For the elongation $\bar{\varepsilon}_{11}=3 \%$, the sharp decrease of the electric conductivity can be observed at both $\Phi_{0}=0.17 \mathrm{eV}$ and $\Phi_{0}=0.3 \mathrm{eV}$. However, when the barrier height increases to $0.5 \mathrm{eV}$, the electric conductivity of the insulating composite doesn't vary a lot with the increasing effective strain. This phenomenon gives a view that the polymer matrix would also be taken into account for the material design to obtain the special electro-mehanical function.

\section{Conclusion}

In this paper, we identified a $\mathrm{CZ}$ model using $\mathrm{MD}$ simulations. The $\mathrm{CZ}$ model has enriched a nonlinear mechanical model where graphene sheets are modeled by imperfect 


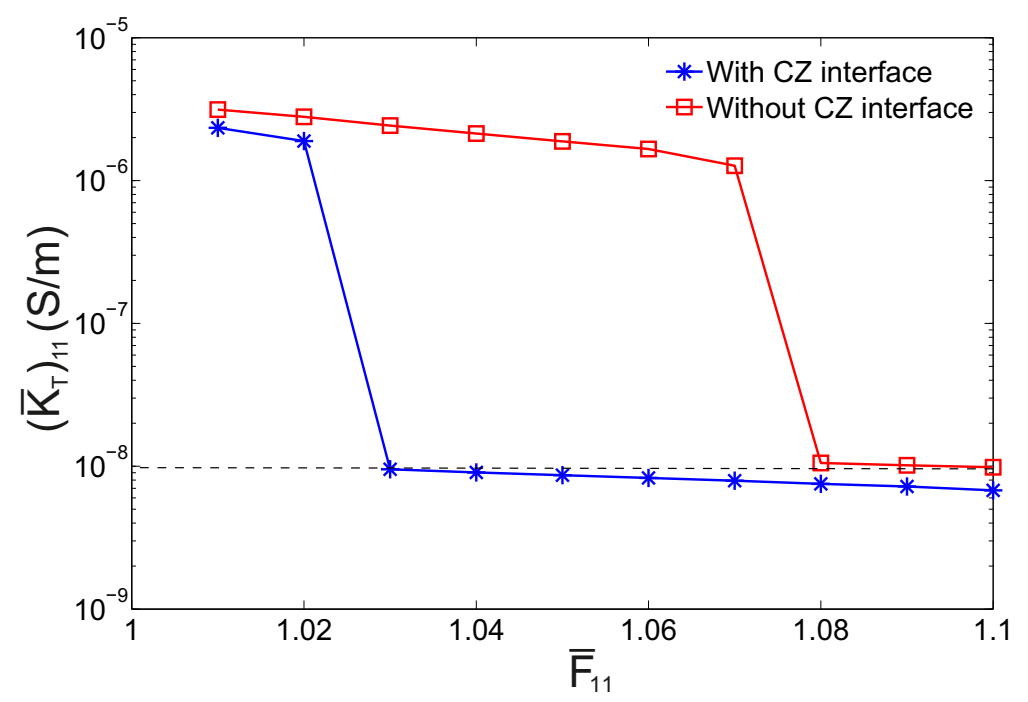

Figure 10. Effective electric conductivity $\left(\overline{K_{T}}\right)_{11}$ as a function of effective strain $\bar{\epsilon}_{11}$ for the composite with 0.66 vol\% graphene both with and without considering the cohesive interface. $\Phi_{0}=0.17$.

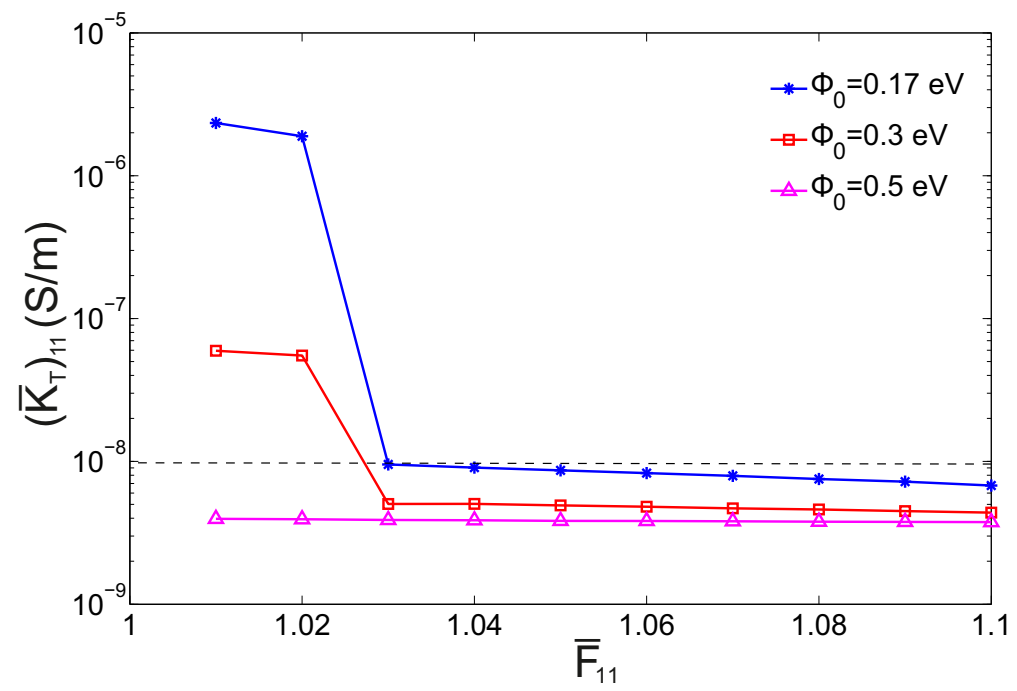

Figure 11. Effective electric conductivity $\left(\overline{K_{T}}\right)_{11}$ as a function of effective strain $\bar{\epsilon}_{11}$ for the composite with $0.66 \mathrm{vol} \%$ graphene at barrier height $\Phi_{0}=0.17,0.3$ and $0.5 \mathrm{eV}$ respectively.

interfaces, i.e. a combination of an elastic membrane and a CZ model. Finally, the mechanical model allowed us to generate deformed RVEs to study influence of strain and interfacial decohesion on the conductivity of graphene/polymer nanocomposites. An electric continuum model, that incorporates the tunneling effect, have been used to study the effective conductivity and the influence of the macroscopic elongation, the interfacial decohesion and the potential barrier height. 
This multiscale and multiphysics approach has shown the existence of a piezoresistive effect for graphene/polymer nanocomposites. This effect is very significant for graphene volume fraction just above the percolation threshold because a conductorinsulator transition is observed for elongations above $2 \%$. In addition, the model has demonstrated the importance of decohesion on the conductor-insulator transition. Indeed, the transition appears for an elongation of $8 \%$ instead of $2 \%$, when the interfacial decohesion is removed in the mechanical model. It is interesting to note that this strategy could be used to model and design damage detection sensors $[152,153]$.

\section{Aknowledgements}

J. Yvonnet thanks the financial support of Institut Universitaire de France (IUF). X. Lu thanks the financial support of China Scholarship Council(CSC) for the Ph.D. thesis. The molecular simulations was carried out using the LAMMPS molecular dynamics software [154]. The GMSH mesh generator are used to create all meshes [155].

\section{References}

[1] Stankovich S, Dikin D, Dommett G, Kohlhaas K, Zimney E, Stach E, Piner R, Nguyen S and Ruoff R 2006 Nature 442 282-286

[2] Song Z, Xu T, Gordin M, Jiang Y, Bae I, Xiao Q, Zhan H, Liu J and Wang D 2012 Nano Lett. $122205-2211$

[3] Liu N, Luo F, Wu H, Liu Y, Zhang C and Chen J 2008 Adv. Funct. Mater. 18 1518-1525

[4] Steurer P, Wissert R, Thomann R and Mülhaupt R 2009 Macromol. Rapid Commun. 30 316-327

[5] Verdejo R, Bernal M, Romasanta L and Lopez-Manchado M 2011 J. Mater. Chem. 21 3301-3310

[6] Potts J R, Dreyer D R, Bielawski C W and Ruoff R S 2011 Polymer 52 5-25

[7] Galpaya D, Wang M, Liu M, Motta N, Waclawik E and Yan C 2012 Graphene 1 30-49

[8] Liang J, Huang Y, Zhang L, Wang Y, Ma Y, Guo T and Chen Y 2009 Adv. Funct. Mater. 19 $2297-2302$

[9] Rafiee M, Rafiee J, Wang Z, Song H, Yu Z and Koratkar N 2009 ACS Nano 3 3884-3890

[10] Rafiee M, Rafiee J, Srivastava I, Wang Z, Song H, Yu Z and Koratkar N 2010 Small 6 179-183

[11] Zhao X, Zhang Q, Chen D and Lu P 2010 Macromolecules 43 2357-2363

[12] Li W, Dichiara A and Bai J 2013 Compos. Sci. Technol. 74 221-227

[13] Kang I, Heung Y Y, Kim J H, Lee J W, Gollapudi R, Subramaniam S, Narasimhadevara S, Hurd D, Kirikera G R, Shanov V, Schulz M J, Shi D, Boerio J, Mall S and Ruggles-Wren M 2006 Compos. Part B-Eng. 37 382-394

[14] Huang X, Yin Z, Wu S, Qi X, He Q, Zhang Q, Yan Q, Boey F and Zhang H 2011 Small 7 $1876-1902$

[15] Sadasivuni K K, Ponnamma D, Kim J and Thomas S (eds) 2015 Graphene-based polymer nanocomposites in electronics (Springer)

[16] Xu J, Wang K, Zu S Z, Han B H and Wei Z 2010 ACS Nano 4 5019-5026

[17] Fu X W, Liao Z M, Zhou J X, Zhou Y B, Wu H C, Zhang R, Jing G, Xu J, Wu X, Guo W et al. 2011 Appl. Phys. Lett. 99213107

[18] Frank O, Tsoukleri G, Riaz I, Papagelis K, Parthenios J, Ferrari A C, Geim A K, Novoselov K S and Galiotis C 2011 Nat. Commun. 2255

[19] Eswaraiah V, Balasubramaniam K and Ramaprabhu S 2011 J. Mater. Chem. 21 12626-12628

[20] Eswaraiah V, Balasubramaniam K and Ramaprabhu S 2012 Nanoscale 4 1258-1262

[21] Huang Y, Liang J and Chen Y 2012 Small 8 1805-1834 
[22] Hwang S H, Park H W, Park Y B, Um M K, Byun J H and Kwon S 2013 Compos. Sci. Technol. $891-9$

[23] Tamburrano A, Sarasini F, De Bellis G, D'Aloia A and Sarto M 2013 Nanotechnology 24465702

[24] Boland C S, Khan U, Ryan G, Barwich S, Charifou R, Harvey A, Backes C, Li Z, Ferreira M S, Möbius M E et al. 2016 Science 354 1257-1260

[25] Awasthi A, Lagoudas D and Hammerand D 2008 Modell. Simul. Mater. Sci. Eng. 17015002

[26] Lu X, Yvonnet J, Detrez F and Bai J 2017 J. Comput. Phys. 337 116-131

[27] Lu X, Yvonnet J, Detrez F and Bai J 2018 J. Compos. Mater. 52 2767-2775

[28] Allaoui A, Hoa S and Pugh M 2008 Compos. Sci. Technol. $68410-416$

[29] Martin-Gallego M, Bernal M, Hernandez M, Verdejo R and Mópez-Manchado M 2013 Eur. Polym. J. 49 1347-1353

[30] Zeng X, Xu X, Shenaiand P M, E Kovalev E, Baudot C, Mathews N and Zhao Y 2011 J. Phys. Chem. C 115 21685-21690

[31] Otten R H and van der Schoot P 2009 Phys Rev Lett 103225704

[32] Ambrosetti G, Grimaldi C, Balberg I, Maeder T, Danani A and Ryser P 2010 Phys. Rev. B 81 155434

[33] Otten R H and van der Schoot P 2011 J. Chem. Phys. 134094902

[34] Wang Y, Weng G J, Meguid S A and Hamouda A M 2014 J. Appl. Phys. 115193706

[35] Wang Y, Shan J W and Weng G J 2015 J. Appl. Phys. 118065101

[36] Hashemi R and Weng G J 2016 Carbon 96 474-490

[37] Xia X, Zhong Z and Weng G J 2017 Mech. Mater. 109 42-50

[38] Xia X, Wang Y, Zhong Z and Weng G J 2017 Carbon 111 221-230

[39] Manta A, Gresil M and Soutis C 2017 Appl. Compos. Mater. 24 281-300

[40] Bövik P 1994 Q. J. Mech. Appl. Math 47 17-42

[41] Gurtin M, Weissmüller J and Larche F 1998 Philos. Mag. A 78 1093-1109

[42] Benveniste Y and Miloh T 2001 Mech. Mater. 33 309-323

[43] Hashin Z 2002 J. Mech. Phys. Solids 50 2509-2537

[44] Benveniste Y 2006 J. Mech. Phys. Solids 54 708-734

[45] Monchiet V and Bonnet G 2010 Int. J. Eng. Sci. 48 1762-1768

[46] Gu S, Monteiro E and He Q C 2011 Compos. Sci. Technol. 71 1209-1216

[47] Gu S and He Q C 2011 J. Mech. Phys. Solids 59 1413-1426

[48] Gu S T, Liu J T and He Q C 2014 Int. J. Solids Struct. 51 2283-2296

[49] Chatzigeorgiou G, Meraghni F and Javili A 2017 J. Mech. Phys. Solids 106 257-282

[50] Javili A, Steinmann P and Mosler J 2017 Comput. Methods Appl. Mech. Engrg. 317 274-317

[51] Javili A 2018 Math. Mech. Solids 1081286518766980

[52] Gurtin M E and Murdoch A I 1975 Arch. Ration. Mech. An. 57 291-323

[53] Moeckel G 1975 Arch. Ration. Mech. An. 57 255-280

[54] Murdoch A I 1976 Q. J. Mech. Appl. Math 29 245-275

[55] Daher N and Maugin G 1986 Acta Mech. 60 217-240

[56] Dell'Isola F and Romano A 1987 Int. J. Eng. Sci. 25 1459-1468

[57] Steigmann D and Ogden R 1999 P. Roy. Soc. Lond. A Mat. 455 437-474

[58] Fried E and Todres R E 2005 J. Elasticity 80 97-151

[59] Huang Z and Wang J X 2006 Acta Mech. 182 195-210

[60] Fried E and Gurtin M E 2007 Continuum Mech. Therm. 19 253-271

[61] Steinmann P 2008 J. Mech. Phys. Solids 56 772-800

[62] Saeb S, Steinmann P and Javili A 2018 Philos. Mag. 98 437-463

[63] Barenblatt G I 1959 J. Appl. Math. Mech.-USS 23 622-636

[64] Dugdale D S 1960 J. Mech. Phys. Solids 8 100-104

[65] Barenblatt G I 1962 Adv. Appl. Mech. 7 55-129

[66] Miller R E and Shenoy V B 2000 Nanotechnology 11139

[67] Shenoy V B 2002 Int. J. Solids Struct. 39 4039-4052 
[68] Sharma P, Ganti S and Bhate N 2003 Appl. Phys. Lett. 82 535-537

[69] Sharma P and Ganti S 2004 J. Appl. Mech. 71 663-671

[70] Duan H, Wang J x, Huang Z and Karihaloo B L 2005 J. Mech. Phys. Solids 53 1574-1596

[71] Gao W, Yu S and Huang G 2006 Nanotechnology 171118

[72] Le Quang H and He Q C 2007 J. Mech. Phys. Solids 55 1899-1931

[73] Park H S and Klein P A 2008 J. Mech. Phys. Solids 56 3144-3166

[74] Yvonnet J, Quang H L and He Q C 2008 Comp. Mech. 42 119-131

[75] Chhapadia P, Mohammadi P and Sharma P 2011 J. Mech. Phys. Solids 59 2103-2115

[76] Monteiro E, He Q C and Yvonnet J 2011 Int. J. Eng. Sci. 49 985-1000

[77] Javili A, McBride A, Mergheim J, Steinmann P and Schmidt U 2013 Int. J. Solids Struct. 50 2561-2572

[78] Xu X P and Needleman A 1994 J. Mech. Phys. Solids 42 1397-1434

[79] Ortiz M and Pandolfi A 1999 Int. J. Numer. Meth. Engng. 44 1267-1282

[80] Tijssens M G, Sluys B L and van der Giessen E 2000 Eur. J. Mech. A-Solid. 19 761-780

[81] Alfano G and Crisfield M 2001 Int. J. Numer. Meth. Engng. 50 1701-1736

[82] Klein P, Foulk J, Chen E, Wimmer S and Gao H 2001 Theor. Appl. Fract. Mech. 37 99-166

[83] Abdul-Baqi A and Van der Giessen E 2001 Thin Solid Films 381 143-154

[84] Abdul-Baqi A and Van der Giessen E 2002 Int. J. Solids Struct. 39 1427-1442

[85] Gao H and Ji B 2003 Eng. Fract. Mech. 70 1777-1791

[86] Van den Bosch M, Schreurs P and Geers M 2006 Eng. Fract. Mech. 73 1220-1234

[87] González C and LLorca J 2007 Compos. Sci. Technol. 67 2795-2806

[88] Park K, Paulino G H and Roesler J R 2009 J. Mech. Phys. Solids 57 891-908

[89] Bhandakkar T K, Chng A C, Curtin W and Gao H 2010 J. Mech. Phys. Solids 58 530-541

[90] Park K and Paulino G H 2011 Appl. Mech. Rev. 64060802

[91] Mosler J and Scheider I 2011 J. Mech. Phys. Solids 59 1647-1668

[92] Shishvan S S and Van der Giessen E 2013 Modell. Simul. Mater. Sci. Eng. 21065006

[93] Qian J, Lin J, Xu G K, Lin Y and Gao H 2017 J. Mech. Phys. Solids 101 197-208

[94] Segurado J and LLorca J 2004 Int. J. Solids Struct. 41 2977-2993

[95] Jiang L Y, Huang Y, Jiang H, Ravichandran G, Gao H, Hwang K and Liu B 2006 J. Mech. Phys. Solids $\mathbf{5 4} 2436-2452$

[96] Yao H and Gao H 2007 Int. J. Solids Struct. 44 8177-8193

[97] Van den Bosch M, Schreurs P and Geers M 2007 Eur. J. Mech. A-Solid. 26 1-19

[98] Bhandakkar T K and Gao H 2010 Int. J. Solids Struct. 47 1424-1434

[99] Charles Y, Estevez R, Bréchet Y and Maire E 2010 Eng. Fract. Mech. 77 705-718

[100] Williams J, Segurado J, LLorca J and Chawla N 2012 Mat. Sci. Eng. A-Struct. $557113-118$

[101] Tijssens M, Van der Giessen E and Sluys L 2000 Int. J. Solids Struct. 37 7307-7327

[102] Tijssens M, Van der Giessen E and Sluys L 2000 Mech. Mater. 32 19-35

[103] Estevez R, Tijssens M and Van der Giessen E 2000 J. Mech. Phys. Solids 48 2585-2617

[104] Estevez R and Van der Giessen E 2005 Modeling and computational analysis of fracture of glassy polymers Intrinsic Molecular Mobility and Toughness of Polymers II (Springer) pp 195-234

[105] Rottler J, Barsky S and Robbins M O 2002 Phys. Rev. Lett. 89148304

[106] Rottler J and Robbins M O 2002 Phys. Rev. Lett. 89195501

[107] Rottler J and Robbins M O 2003 Phys. Rev. E 68011801

[108] Rottler J 2009 J. Phys.: Condens. Matter 21463101

[109] Liao K and Li S 2001 Appl. Phys. Lett. 79 4225-4227

[110] Gou J, Minaie B, Wang B, Liang Z and Zhang C 2004 Comp. Mater. Sci. 31 225-236

[111] Chowdhury S and Okabe T 2007 Compos. Part A-Appl. S. 38 747-754

[112] Li C, Browning A R, Christensen S and Strachan A 2012 Compos. Part A-Appl. S. 43 1293-1300

[113] Rahman R and Haque A 2013 Compos. Part B-Eng. 54 353-364

[114] Spearot D E, Jacob K I and McDowell D L 2004 Mech. Mater. 36 825-847

[115] Yamakov V, Saether E, Phillips D R and Glaessgen E H 2006 J. Mech. Phys. Solids 54 1899-1928 
[116] Liu X, Li S and Sheng N 2008 Comp. Mech. 42 543-553

[117] Zeng X and Li S 2010 Comput. Methods Appl. Mech. Engrg. 199 547-556

[118] Paggi M and Wriggers P 2011 Comp. Mater. Sci. 50 1625-1633

[119] Paliwal B and Cherkaoui M 2013 Int. J. Solids Struct. 50 3346-3360

[120] Hoang M, Yvonnet J, Mitrushchenkov A, Chambaud G and Duan H 2015 Nanotechnology 26 115703

[121] Namilae S and Chandra N 2005 J. Eng. Mater.-T ASME 127 222-232

[122] Lu W, Wu J, Jiang L, Huang Y, Hwang K and Liu B 2007 Philos. Mag. 87 2221-2232

[123] Liu Y, Nishimura N, Qian D, Adachi N, Otani Y and Mokashi V 2008 Eng. Anal. Bound. Elem. 32 299-308

[124] Chen Y, Chia J, Su Z, Tay T and Tan V 2013 Polymer 54 766-773

[125] Song S, Chen Y, Su Z, Quan C and Tan V B 2014 Compos. Sci. Technol. $100189-197$

[126] Paliwal B, Lawrimore W B, Chandler M Q and Horstemeyer M F 2017 Philos. Mag. 97 1179-1208

[127] Shenoy V B 2005 Phys. Rev. B 71094104

[128] Park H S, Klein P A and Wagner G J 2006 Int. J. Numer. Meth. Engng. 68 1072-1095

[129] Yvonnet J, Mitrushchenkov A, Chambaud G and He Q C 2011 Comput. Methods Appl. Mech. Engrg. 200 614-625

[130] Yvonnet J, Mitrushchenkov A, Chambaud G, He Q C and Gu S T 2012 J. Appl. Phys. 111 124305

[131] Davydov D, Javili A and Steinmann P 2013 Comp. Mater. Sci. 69 510-519

[132] Davydov D, Javili A, Steinmann P and McBride A 2013 A comparison of atomistic and surface enhanced continuum approaches at finite temperature Surface Effects in Solid Mechanics (Springer) pp 43-57

[133] Elsner B, Müller S, Bargmann S and Weissmüller J 2017 Acta Materialia 124 $468-477$

[134] Mayo S L, Olafson B D and Goddard W A 1990 J. Phys. Chem. 94 8897-8909

[135] Binder K 1995 Monte Carlo and molecular dynamics simulations in polymer science (Oxford University Press)

[136] Gao J 1995 J. Chem. Phys. 102 1074-1077

[137] Perez M, Lame O, Leonforte F and Barrat J L 2008 J. Chem. Phys. 128234904

[138] Berendsen H J C, Postma J P M, van Gunsteren W F, DiNola A and Haak J R 1984 J. Chem. Phys. 81 3684-3690

[139] Martyna G J, Tobias D J and Klein M L 1994 J. Chem. Phys. 101 4177-4189

[140] Shinoda W, Shiga M and Mikami M 2004 Phys. Rev. B 69134103

[141] Thomas C, Seguela R, Detrez F, Miri V and Vanmansart C 2009 Polymer $503714-3723$

[142] Detrez F 2008 Nanomécanismes de déformation des polymères semi-cristallins: étude in situ par microscopie à force atomique et modélisation. Ph.D. thesis Université Lille I

[143] Kramer E J and Berger L L 1990 Fundamental processes of craze growth and fracture Crazing in Polymers Vol. 2 (Springer) pp 1-68

[144] Holzapfel G 2000 Nonlinear Solid Mechanics A continuum approach for engineering (Wiley)

[145] Truesdell C and Noll W 2004 The Non-Linear Field Theories of Mechanics (Springer)

[146] Gurtin M E, Fried E and Anand L 2010 The Mechanics and Thermodynamics of Continua (Cambridge)

[147] Tadmor E, Miller R and Elliott R 2012 Continuum Mechanics and Thermodynamics From Fundamental Concepts to Governing Equations (Cambridge)

[148] Theodorou D N and Suter U W 1986 Macromolecules 19 139-154

[149] Lu X 2017 Multiscale electro-mechanical modeling of graphene/polymer nanocomposites Ph.D. thesis Université Paris-Saclay

[150] Yvonnet J, He Q C and Toulemonde C 2008 Compos. Sci. Technol. 68 2818-2825

[151] Simmons J G 1963 J. Appl. Phys. 34 2581-2590

[152] Flandin L, Brechet Y and Cavaille J Y 2001 Compos. Sci. Technol. 61 895-901

[153] Xia Z H and Curtin W A 2007 Compos. Sci. Technol. 67 1518-1529 
[154] Plimpton S 1995 J. Comput. Phys. 117 1-19

[155] Geuzaine C and Remacle J F 2009 Int. J. Numer. Methods Eng. 79 1309-1331

[156] Krauth W 2006 Statistical mechanics: algorithms and computations vol 13 (OUP Oxford)

[157] Torquato S 2013 Random heterogeneous materials: microstructure and macroscopic properties vol 16 (Springer Science \& Business Media)

\section{Appendix A. RVE generation for graphene/polymer nanocomposite}

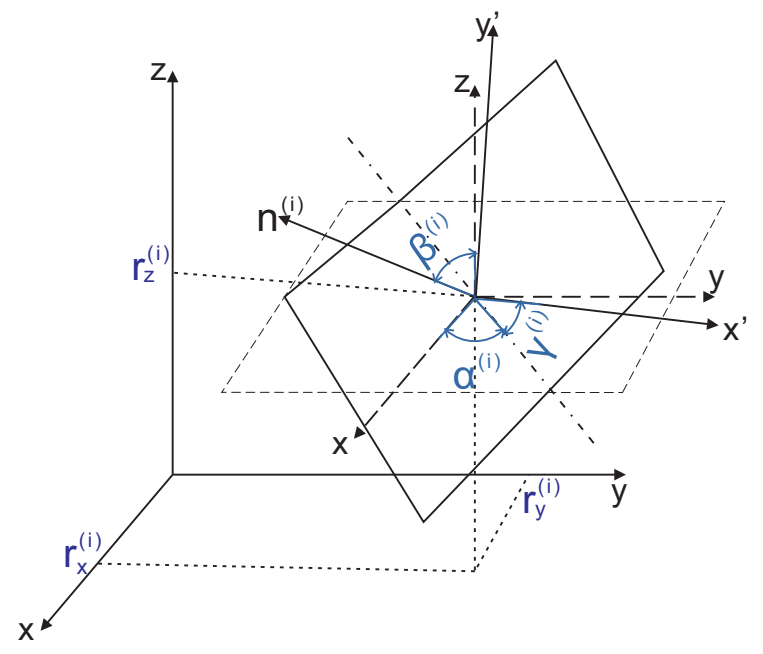

Figure A1. Scheme of the position and oriention of graphene platelets in the 3D space.

The position and orientation of $i$ th graphene sheet in RVE are defined by 6 degrees of freedom $\left\{r_{x}^{(i)}, r_{y}^{(i)}, r_{z}^{(i)}, \alpha^{(i)}, \beta^{(i)}, \gamma^{(i)}\right\}$ where $r_{x}^{(i)}, r_{y}^{(i)}, r_{z}^{(i)}$ are the coordinates of the center of graphene sheet, and $\alpha^{(i)}, \beta^{(i)}, \gamma^{(i)}$ are the Euler angles. Note that $\alpha^{(i)}, \beta^{(i)}$ give the orientation of the unit normal of graphene sheet, $n^{(i)}$, and $\gamma^{(i)}$ gives the orientation of graphene sheet around the unit normal $n^{(i)}$ (see Figure A1).

The different microstructures are generated by a Markov-chain with hard-inclusion Metropolis algorithm (see $[156,157]$ for review in the case hard-spheres). First, an initial configuration of the system is chosen such the graphene sheet centers are on cubic lattice with random orientation and without overlapping. Then, one randomly chosen graphene sheet is attempted to move for each step of Metropolis algorithm. A new position for the graphene sheet under consideration is chosen by six random parameters: $(\Delta x, \Delta y, \Delta z) \in\left[-\delta_{x}, \delta_{x}\right]^{3}, \Delta \alpha \in[-\pi, \pi], \Delta \beta \in\left[-\delta_{\beta}, \delta_{\beta}\right]$ and $\Delta \gamma \in\left[-\delta_{\gamma}, \delta_{\gamma}\right]$. The parameter $(\Delta x, \Delta y, \Delta z, \Delta \gamma)$ are generated with an uniform distribution over their definition domains and the couple of increment angles $(\Delta \alpha, \Delta \beta)$ are generate with an uniform distrution on the part of the unit sphere defined by $\delta_{\beta}$. The new position of graphene sheet is then $\left(x^{(i)}+\Delta x, y^{(i)}+\Delta y, z^{(i)}+\Delta z, \alpha^{(i)}+\Delta \alpha, \beta^{(i)}+\Delta \beta, \gamma^{(i)}+\Delta \gamma\right)$. The parameters $\delta_{x}, \delta_{\beta}$ and $\delta_{\gamma}$ are adjusted to give about $50 \%$ acceptance of the new position. Periodic boundary conditions are employed throughout the simulation. 
Therefore, the acceptance step of this Metropolis algorithm reduces to checking that this displacement does not cause overlap between graphene sheets. To control the overlapping, the graphene sheets are discretized by a compact centered square lattice of small fictitious spherical particles with radius $\delta$. The non-overlapping condition is

$$
r_{i j}^{2} \geq \delta^{2} \quad \forall i, j
$$

where $r_{i j}$ is a distance between the fictitious spherical particles $i$ and $j$.

To generate a series of independent isotropic RVE samples as random maps, the position and orientation are saved during a Markov-chain sampling with regular interval to ensure the statistic independence of two RVEs. Note that the first RVE is saved when the orientation of the unit normal of graphene sheets is isotropic in an average sense. 